\title{
Review of: "Changes in adiposity, physical activity, cardiometabolic risk factors, diet, physical capacity and well-being in inactive women and men aged 57- 74 years with obesity and cardiovascular risk - A 6- month complex lifestyle intervention with 6-month follow-up"
}

\section{Górnicka Magdalena}

Potential competing interests: The author(s) declared that no potential competing interests exist.

It is an interesting subject and results, but I have great doubts about the number of participants, results and conclusions.

I am aware of the difficulty of maintaining the group with long-term intervention, so I suggest adding "a pilot study" to the title. I would ask you to define the term "inactive" and to use the correct terminology consistently. "Physical inactivity: is not the same as" insufficient physical activity ". Please add used criteria to assess 'inactivity'.

Introduction: it should be improved; consider more cardiometabolic risk factor than PA.

Materials and methods: You wrote that you assessed BW, BMI, WC, but there are no criteria for assessing adiposity. In addition, obesity or adiposity are included in the cardiometabolic risk factors in addition to those included in the manuscript. I propose to combine these two sections and clearly define the criteria used.

Please clarify in which way you assessed nutritional values based on data from FFQ (the question: How often did you eat....? and answer e.g. two times a day - doesn't allow to estimate energy and nutrients content in diet). In cited reference (24) the semi-quantitative FFQ, included 270 food items was used. Please specify whether it was the same questionnaire in full.

Results: Please show the discussed changes also separately for men and women (sex-differences e.g in body fat). Table 2- please clarify: body fat mass/body lean mass as \%?Figure 2-4 move to Supporting information, and the descrption (page 9; under Fig. 2) add to Figures.

I suggest to order the results and show for all outcomes values for baseline, end of intervention and end of follow-up. I could not find clear results consistent with the title and purpose of the study, therefore I cannot judge the discussions and conclusions. In addition, in the title you described that your study considered women and men aged 57-74 years with obesity and cardiovascular risk, so conclusions should also take into account this specificity. 
